\title{
El comercio justo en la opinión pública española. Perfiles y orientaciones
}

\section{Ramón Llopis Goig*}

Universidad de Valencia. Departamento de Sociología y Antropología Social Avda. dels Tarongers, s/n (Edificio Oriental). 46022 Valencia ramon.llopis@uv.es

\section{Resumen}

Durante los últimos años, la sociología del consumo ha sugerido el surgimiento de un sentido de la "ciudadanía a través del consumo». Este planteamiento se ha apoyado en la aparición y el desarrollo de propuestas alternativas como el comercio justo (junto a otras como el consumo responsable, el comercio de reciclados o el desarrollo sostenible), así como en la emergencia de los denominados «nuevos consumidores», caracterizados por valores de naturaleza más reflexiva como la solidaridad, la responsabilidad social, el multiculturalismo y la ecología. A partir de los datos contenidos en el estudio 2419 del Centro de Investigaciones Sociológicas (CIS) del año 2001, el trabajo que presentamos se ha planteado, en primer lugar, estudiar las características sociodemográficas de la población consumidora de productos de comercio justo, una tarea prácticamente inédita para la ciencia social española, pese a las dos décadas de antigüedad de este movimiento en nuestro contexto. En segundo lugar, sometemos a prueba empírica la habitualmente aceptada «orientación global» de los consumidores de productos de comercio justo.

Palabras clave: comercio justo, consumo, orientación global.

\section{Abstract. Fair trade in Spanish public opinion. Profiles and orientations}

In the last few years Sociology of Consumerism has proposed the emergence of a sense of "citizenship through consumption». This idea has been built up after the creation and development of alternatives such as fair trade (together with responsible consumption, recycled product trade or sustainable development) and the emergence of the so-called «new consumers», with values of a more reflexive nature such as solidarity, social responsibility, multiculturalism and ecology. Starting from the data of the study number 2419 carried out by the Centre of Sociological Investigations (CIS) in 2001, the research that we present has thought about, in the first place, to study consumers of trade fair products profile, a practically unpublished task for the Spanish social science in spite of the two decades of antiquity of this movement in our context. Secondly, we check empirically the habitually accepted "global orientation" of the consumers of fair trade products.

Key words: fair trade, consumption, global orientation. 


\section{Sumario}

$\begin{aligned} \text { 1. Introducción } & \text { 4. Recapitulación y conclusiones } \\ \text { 2. Método } & \text { Bibliografía } \\ \text { 3. Resultados } & \text { Anexo }\end{aligned}$

\section{Introducción}

Aunque el modelo de consumo de masas sigue siendo dominante en las sociedades occidentales, durante la última década se han desarrollado alternativas como el comercio justo, el consumo responsable, el comercio de reciclados o el desarrollo sostenible, que han permitido hablar de la aparición de un sentido de "ciudadanía a través del consumo». En relación con ello, diversos autores (Morace, 1990; Rochefort, 1996; Matathia y Salzman, 2001) han postulado la emergencia de un "nuevo consumidor», alejado tanto del consumidor forjado en la época fordista, como del consumidor representado por la individualización neoliberal y la hiperflexibilización del postfordismo tecnológico y financiero de los años ochenta (Alonso, 2005).

La principal característica de este "nuevo consumidor» sería la incorporación, en su construcción identitaria, de diversos valores de naturaleza más reflexiva, como la solidaridad, el multiculturalismo, la ecología y la aceptación de la diferencia sexual. Esta nueva cultura de consumo podría ser interpretada como una respuesta a la materialización y ampliación de las desigualdades y las exclusiones sociales producidas por los nuevos modelos de consumo; pero también como una reacción a los recientes problemas de seguridad en la cadena alimentaria, el progresivo deterioro ecológico del entorno, o las sospechas cada vez más fundadas sobre las condiciones de producción y comercialización de determinados bienes. Los "nuevos consumidores» se caracterizan por un mayor sentido de la responsabilidad, un mayor interés en la seguridad de los productos, una mayor orientación hacia la información sobre sus ingredientes o condiciones de producción y los efectos sobre la salud y el medio ambiente.

Todo ello ha permitido que se pueda hablar de la aparición de un sentido de "ciudadanía a través del consumo», que convertiría en obsoletos los planteamientos que hacían del consumidor o un ser totalmente alienado o un racional calculador de sus propios intereses. Los denominados «nuevos consumidores» se comportan de un modo más reflexivo, hacen gala de un mayor autocontrol y están desarrollando un modelo de "consumidor ciudadano", que se aleja de cualquier proyecto de transformación política radical, pero también de la legitimación de una sociedad de consumo con la que ya se muestran críticos (Alonso, 2005). Junto a otras propuestas, como el consumo responsable, el comercio de reciclados o el desarrollo sostenible, un ejemplo de estas alternativas en las formas de consumir y vivir la constituye el "comercio justo".

La expresión "comercio justo» es la denominación habitual para referirse a un sistema alternativo de relaciones comerciales entre los países productores 
del Sur y los países consumidores del Norte. Es una práctica que promueve cambios sociales y productivos tanto en el Sur como en el Norte, ya que no sólo actúa sobre las transacciones comerciales, sino también sobre los hábitos de consumo de los ciudadanos ${ }^{1}$. Los principales objetivos del comercio justo son garantizar un salario justo a los trabajadores, mejorar las condiciones de seguridad e higiene del lugar de trabajo, fomentar la igualdad de oportunidades para las mujeres, proteger los derechos infantiles, salvaguardar las minorías étnicas y preservar el medio ambiente (Solé, 2003: 34). Así, aunque inicialmente se desarrolló como un medio de ofrecer una salida comercial al resultado del trabajo de ciertos grupos de productores de los países más desfavorecidos y como una forma de promover un consumo responsable entre los consumidores de los países del Norte; con posterioridad, el comercio justo ha asumido prácticas de denuncia y presión política a determinadas prácticas empresariales y políticas comerciales discriminatorias (Cantos, 1998: 101) ${ }^{2}$.

En España, el comercio justo surgió en 1986, cuando, en el País Vasco, aparece la ONG los Traperos de Emaús, y en Andalucía, la Cooperativa Sandino, en la actualidad denominada Iniciativas de Economía Alternativa y Solidaria (IDEAS). El movimiento se difundió por el resto de España a través de organizaciones como Equimercado o Alternativa 3. Estas iniciativas desencadenaron la incorporación de otras ONG, como Intermón, Setem o Sodepaz, tras lo cual algunas de ellas iniciaron labores de importación y distribución. En 1995 se formó la Red de Economía Alternativa y Solidaria (REAS), compuesta por veintiocho asociaciones, importadoras y distribuidoras, de carácter nacional e internacional. En 1996 nació la Coordinadora Estatal de Organizaciones de Comercio Justo, integrada por veintiséis entidades presentes en once comunidades autónomas (Solé, 2003: 43).

Según los datos contenidos en el primer anuario de comercio justo realizado en nuestro país (Setem, 2004), en el año 2003, las ventas totales de productos de comercio justo alcanzaban los 10.662 .210 euros, de las cuales un $72,2 \%$ se concentraba en el canal de distribución minorista. Esa cifra suponía un incremento del 19\% respecto a los 8.975.817 euros del año 2002. Un año antes, en 2001, el volumen de ventas de productos de comercio justo en España era de 7.220.000 euros, una cifra que equivalía al 1,9\% del volumen total del sector en Europa (EFTA, 2001) $)^{3}$. Por tipología, los productos de artesanía suponían

1. De hecho, diversos trabajos sobre comercio justo señalan una triple dimensión esencial en éste, puesto que hacen alusión a la comercialización, sensibilización y denuncia (Cantos, 1998: 96; Setem, 2004: 13).

2. Para más detalles sobre aspectos relativos al nacimiento y al desarrollo del comercio justo, así como a definiciones, experiencias, modelos y propuestas doctrinales, véase MartínezOrozco (1996), Cantos (1998), Barrat (1998), Hinojosa (2002), Setem (1999, 2004), Solé (2003) y Montagut y Vivas (2006).

3. Cifras muy superiores correspondían a los países con mayor volumen: Alemania (101.100.000 euros, que suponían un 27,3\% del total europeo), Holanda (71.500.000 euros, un 19,3\%), Reino Unido (69.600.000 euros, 18,8\%) y Suiza (51.000.000 euros, 13,8\%) (Setem, 2004: 40). 
4.999.020 euros en 2003, mientras los de alimentación alcanzaban los 5.256.720. Entre éstos últimos, el café suponía un 21,2\% del total de las ventas; el cacao, un $11,1 \%$, y el azúcar, un 5,4\%. Entre los productos de artesanía, los de decoración eran un $11,9 \%$ y la ropa un $8,1 \%$ (Setem, 2004).

La realidad socioeconómica que muestran estos datos contrasta, sin embargo, con la escasa atención que esta nueva modalidad de consumo ha recibido de los científicos sociales españoles. Pese a las más de dos décadas de antigüedad del comercio justo en España, sorprende la escasa atención que aquéllos le han prestado ${ }^{4}$. Así, mientras resulta fácil encontrar trabajos, realizados generalmente por parte de directivos o técnicos de alguna organización implicada en este movimiento, que abordan el comercio justo desde una perspectiva teórica o doctrinal, los trabajos realizados desde una perspectiva científica son prácticamente inexistentes, algo que no se entiende teniendo en cuenta el volumen económico que ya supone y el interés que como fenómeno social puede tener para las ciencias sociales 5 .

Una excepción a esta situación la constituye la información contenida en el barómetro del CIS mencionado anteriormente ${ }^{6}$. Esta encuesta permite constatar que el porcentaje de españoles que conoce o ha oído hablar del comercio justo como alternativa de compra se sitúa en el 35,9\% de la población mayor de edad. Sin embargo, cuando en esa misma encuesta, previamente, se preguntó por el conocimiento del comercio justo como una nueva modalidad de campañas desarrolladas por las ONG, el porcentaje fue ligeramente inferior y se situó en el 33,2\%. Este grado de conocimiento es claramente inferior al que estudios similares han registrado en Francia (91\%), Holanda (74\%) o Suiza (60\%); y se muestra más próximo a los datos disponibles sobre Alemania (41\%), Bélgica (36\%), Dinamarca (36\%) y Austria (30\%). Porcentajes inferiores a España serían los de Suecia (13\%) y Noruega $(6 \%)^{7}$.

La citada encuesta permite, además, constatar que un $44,9 \%$ de las personas que ha oído hablar del comercio justo ha comprado en alguna ocasión productos de este tipo. Referido al conjunto de la población española mayor de edad, la cifra de personas que habrían adquirido productos de comercio justo alcanzaría el 16,1\%. Por otro lado, al preguntar por la posible evolución futura del comercio justo, un 5,6\% de los encuestados opinó que éste constituirá la base del comercio mundial; un 48,5\%, que será una forma más de comportamiento del consumidor, y un $11,6 \%$, que es una moda que desaparecerá. El restante 34,3\% no supo qué responder.

4. Como han señalado recientemente Carraro, Fernández y Verdú (2006: 123).

5. Habría que destacar los recientes trabajos del Institut Català de Consum, Els catalans i el comerç just $i$ solidari (2004) y la más reciente monografía de Carraro, Fernández y Verdú (2006).

6. Se trata del estudio E-2419, realizado por el Centro de Investigaciones Sociológicas entre el 22 y el 28 de mayo de 2001. Hasta donde llega nuestro conocimiento, aún no se ha publicado ningún trabajo que explote la información relativa a comercio justo contenida en esa encuesta.

7. Datos extraídos de Fair Trade in Europe 2001, coordinado por Jean-Marie Krier (2001: 71-73). 
Este artículo pretende profundizar en el estudio del comercio justo en la sociedad española a partir de los datos contenidos en la encuesta del CIS, cuya información básica acabamos de exponer. La investigación que se presenta pretende, en primer lugar, identificar las características sociodemográficas de los ciudadanos españoles que muestran una predisposición al consumo de productos de comercio justo (conocimiento y compra), y, en segundo lugar, probar si, como habitualmente se asume, los consumidores de este tipo de productos son personas caracterizadas por una "orientación global» superior a la del resto de la población. Este segundo objetivo requiere una explicación adicional.

La adquisición y desarrollo de una perspectiva global ha sido subrayada por los principales teóricos de la globalización (Robertson, 1992; Waters, 1995). Según éstos, la globalización está cambiando el aspecto del mundo, pero también nuestra forma de percibirlo. Al hacernos conscientes del proceso globalizador y de sus consecuencias, adoptamos una perspectiva global que se traduce en una apertura a nuevos valores, individuos y grupos, y una expansión del horizonte de posibilidades de acción (Brown, 1995; Giddens, 2001; Keane, 2003). De este modo, la responsabilidad social de los ciudadanos estaría ampliando su horizonte geográfico, que ya no se detendría ante las fronteras nacionales. La investigación que se presenta en este artículo trata de verificar si entre las personas próximas al comercio justo, es decir aquéllas que lo conocen y compran sus productos, puede afirmarse la existencia de una «orientación global». Debe tenerse en cuenta, sin embargo, que este segundo objetivo es abordado de manera exploratoria.

Las características de la encuesta y la estrategia de análisis estadístico aplicada en la investigación se explican en el segundo apartado de este artículo. En el tercero, se exponen los resultados obtenidos mediante la aplicación de diversas técnicas estadísticas multivariables. Por último, se incluye un apartado de discusión y conclusiones donde se examinan las principales implicaciones de los hallazgos obtenidos y se realizan sugerencias para estudios futuros.

\section{Método}

Los datos examinados proceden de una encuesta realizada por el CIS centrada en conocer las opiniones y las actitudes de los españoles hacia las ONG internacionales y el comercio justo, entre otros aspectos. El estudio se basó en una encuesta de 2.493 entrevistas a la población española de ambos sexos de dieciocho y más años. El procedimiento de muestreo fue polietápico, estratificado por conglomerados, con selección de las unidades primarias de muestreo (municipios), de las secundarias (secciones) de forma aleatoria proporcional, y de las unidades últimas (individuos) según rutas aleatorias y cuotas de edad y sexo. El error muestral, para un nivel de confianza del 95,5\% (dos sigmas), un $p=q$, y bajo el supuesto de muestreo aleatorio simple, fue de \pm 2 .

Para abordar los objetivos de esta investigación, se han aplicado diversas técnicas de análisis multivariable. Con el análisis factorial de componentes 
principales, se han extraído dos factores que simplifican y estructuran la información contenida en diversas variables relevantes para el estudio. Tras ello, se ha aplicado el análisis de varianza unidireccional (ANOVA) y la prueba «t» de Student (para las diferencias de medias entre muestras independientes), para comprobar la existencia de diferencias estadísticamente significativas según diversas variables sociodemográficas en los factores previamente obtenidos. Posteriormente, con el análisis de regresión lineal múltiple, se ha examinado la influencia de diversas variables sociodemográficas y actitudinales sobre los factores que componen el comercio justo.

\section{Resultados}

Con el fin de simplificar la información contenida en la encuesta, se aplicó la técnica del análisis factorial a cuatro variables relacionadas con el conocimiento y la compra de productos de comercio justo. Para la extracción de los factores, se ha prescindido de aquéllos con valores propios (eigenvalues) inferiores a la unidad.

El método de extracción ha sido el análisis de componentes principales, y el de rotación la normalización Varimax con Kaiser. El análisis, con un ajuste muestral de 0,536 (Kaiser-Meyer-Olkin Measure of Sampling Adequacy) y significativo al nivel 0,000 (Bartlett's Test of Sphericity), ha arrojado un total de dos factores o dimensiones que explican un $77,6 \%$ de la varianza procedente de la información contenida en las variables introducidas en el análisis.

La tabla 1 muestra las cargas factoriales obtenidas en la matriz rotada para los dos factores resultantes del análisis.

El primer factor se compone de las dos preguntas que exploraban, respectivamente, el grado de conocimiento del comercio justo como alternativa de compra y el conocimiento de las campañas de productos de comercio justo

Tabla 1. Resultados de la matriz rotada del análisis de componentes principales.

\begin{tabular}{lcc}
\hline & $\begin{array}{l}\text { Factor 1: conocimiento } \\
\text { del comercio justo }\end{array}$ & $\begin{array}{l}\text { Factor 2: compra } \\
\text { de productos } \\
\text { de comercio justo }\end{array}$ \\
\hline $\begin{array}{l}\text { Conocimiento del comercio justo } \\
\begin{array}{l}\text { Conocimiento de campañas } \\
\text { de comercio justo }\end{array}\end{array}$ & 0,925 & 0,077 \\
$\begin{array}{l}\text { Ha comprado productos } \\
\text { de comercio justo }\end{array}$ & 0,915 & 0,059 \\
$\begin{array}{l}\text { Ha comprado productos } \\
\text { comercializados por ONG }\end{array}$ & $-0,121$ & 0,862 \\
$\begin{array}{l}\text { Varianza explicada por cada factor } \\
\text { Varianza acumulada total }\end{array}$ & $\begin{array}{l}45,1 \% \\
45,1 \%\end{array}$ & 0,741 \\
\hline & & $32,5 \%$ \\
& $77,6 \%$
\end{tabular}


desarrolladas por las ONG. Este factor, que explica un 45,1\% de la varianza, lo hemos etiquetado con la denominación "conocimiento del comercio justo». En cuanto al segundo factor, aglutina a las restantes dos variables, referidas en este caso a la compra de productos de comercio justo, y a la compra, en general, de productos comercializados por las ONG. Explica un 32,5\% de la varianza y lo hemos denominado "compra de productos de comercio justo".

Las puntuaciones medias en los factores de diversas variables sociodemográficas nos van a permitir realizar un primer análisis del perfil de las personas más próximas al fenómeno del comercio justo, en términos de conocimiento y compra. Para ello, hemos elaborado un gráfico compuesto por un eje horizontal, que recoge las puntuaciones medias en el factor «conocimiento del comercio justo", y un eje vertical, con las puntuaciones medias en el factor "compra de productos de comercio justo".

En el extremo derecho del eje horizontal, aparecen las puntuaciones positivas en el factor "conocimiento del comercio justo", mientras en el extremo izquierdo aparecen las negativas. En cuanto al eje vertical, las puntuaciones positivas aparecen en el polo superior y las negativas, en el inferior.

Tal y como puede verse en el diagrama adjunto, estos dos ejes conforman cuatro espacios diferenciados que permiten situar a los grupos de entrevistados en función de su orientación general hacia el comercio justo. Estos ejes permiten interpretar el gráfico 1.

En el cuadrante A, se sitúan aquellas personas que se caracterizan por tener un mayor conocimiento y comportamiento de compra de productos de comercio justo. En el punto más extremo del cuadrante, se situarían las personas con estudios universitarios. También aparecerían en este cuadrante las personas con estudios de bachillerato o formación profesional, así como aquéllas que, en la actualidad, son estudiantes o se encuentran en situación laboral activa y tienen entre dieciocho y cuarenta y cuatro años.

En el extremo opuesto, en el cuadrante D, se situarían los que tienen las puntuaciones más bajas en ambos factores. Aparecen ahí los jubilados y los pensionistas, las personas con estudios primarios y los mayores de sesenta años.

\begin{tabular}{|c|c|}
\hline $\begin{array}{r}\mathrm{C} \\
\text { Conocimiento - } \\
\text { Compra + }\end{array}$ & $\begin{array}{l}\text { A } \\
\text { Conocimiento + } \\
\text { Compra + }\end{array}$ \\
\hline $\begin{array}{r}\text { D } \\
\text { Conocimiento - } \\
\text { Compra - }\end{array}$ & $\begin{array}{l}\text { B } \\
\text { Conocimiento + } \\
\text { Compra - }\end{array}$ \\
\hline
\end{tabular}




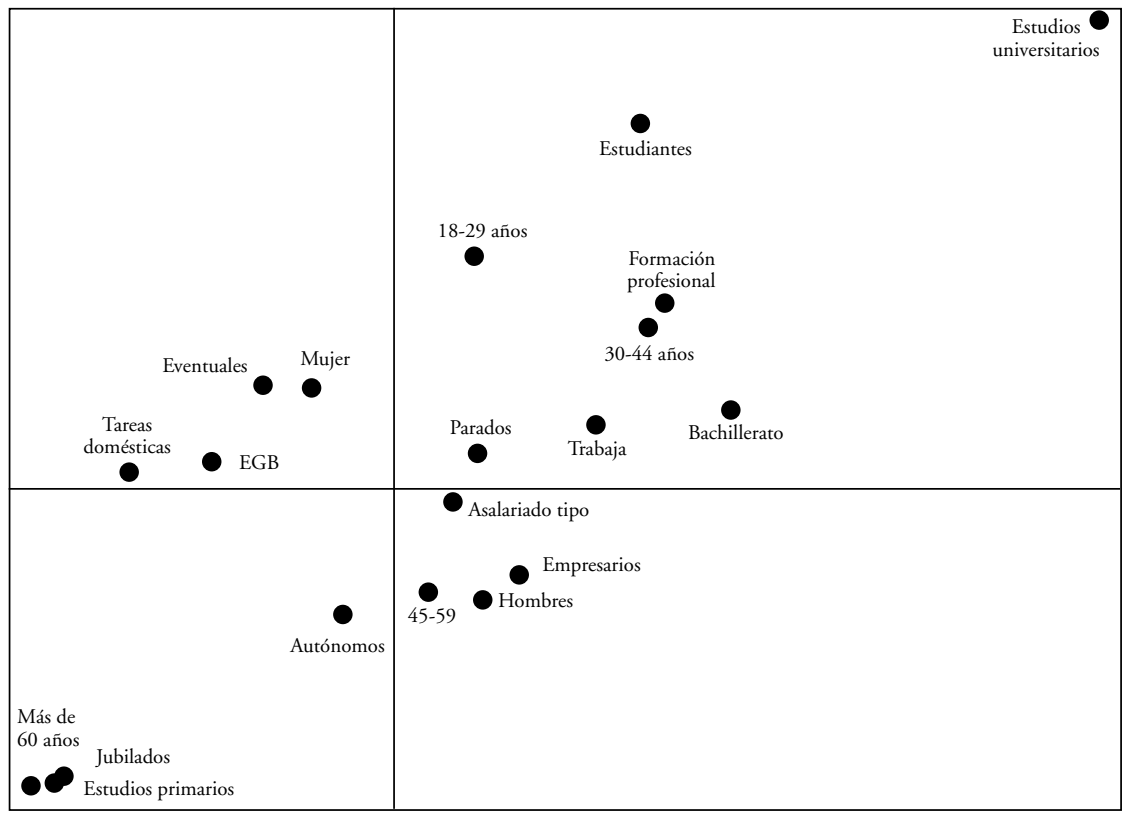

Gráfico 1. Puntuaciones medias en los factores de conocimiento y compra de productos de comercio justo según variables sociodemográficas

Por otro lado, el cuadrante B aglutinaría a aquellos grupos con puntuaciones altas en el factor de conocimiento del comercio justo, pero bajas en relación con la compra de este tipo de productos. Ahí estarían los varones, empresarios y asalariados fijos, con edades entre los cuarenta y cinco y los cincuenta y nueve años.

El cuadrante $\mathrm{C}$, por último, incluiría a los que muestran niveles reducidos de conocimiento pero elevados de compra. Se concentrarían, en este cuadrante, las mujeres, con estudios primarios (educación general básica), con contratos eventuales y dedicación a las tareas domésticas, si bien se sitúan siempre muy cerca de la intersección de los dos ejes.

Ahora bien, más allá del análisis gráfico de las medias factoriales que acabamos de realizar, sería necesario aplicar alguna técnica inferencial que permita identificar la existencia de diferencias estadísticamente significativas entre los distintos grupos poblacionales. Para ello, a continuación, recurriremos al análisis de varianza unidireccional (ANOVA), si bien en el caso del sexo, al tratarse de una variable dicotómica, lo pertinente es aplicar la prueba $t$ de Student para la diferencia de medias de muestras independientes.

La aplicación de la $t$ de Student a las medias de los dos factores según el sexo, muestra que las diferencias entre hombres y mujeres son estadísticamente 
Tabla 2. $T$ de Student para muestras independientes según sexo.

\begin{tabular}{lccc}
\hline Factores & T & GL & Sig. (bilateral) \\
\hline Conocimiento del comercio justo & 4,107 & 2.491 & $0,000^{* * *}$ \\
Compra de productos de comercio justo & $-3,302$ & 2.491 & $0,001^{* *}$ \\
\hline
\end{tabular}

${ }^{* * *}(p<0,001) ;^{* *}(p<0,01) ;^{*}(p<0,05) ;$ ns (no significativo).

Tabla 3. ANOVA unidireccional para los dos factores según edad.

\begin{tabular}{lccccc}
\hline Factores & SC intergrupos & GL & MC & F & P \\
\hline Conocimiento del comercio justo & 127,578 & 3 & 42,526 & 44,767 & $0,000^{* * *}$ \\
Compra de comercio justo & 44,636 & 3 & 14,879 & 15,132 & $0,000^{* * *}$ \\
\hline
\end{tabular}

${ }^{* * *}(p<0,001) ;{ }^{* *}(p<0,01) ;^{*}(p<0,05) ;$ ns (no significativo).

significativas: los hombres puntúan más alto en «conocimiento de productos de comercio justo" $(0,0848$ frente a $-0,0793)$, pero son las mujeres las que obtienen una puntuación más alta en relación con la compra de este tipo de productos $(0,0639 \text { frente a }-0,0683)^{8}$.

En cuanto a la edad, el análisis de varianza muestra que las diferencias según esta variable existentes entre los dos factores son estadísticamente significativas (tabla 3). En el factor de "conocimiento del comercio justo", los mayores de sesenta años se diferencian de manera significativa de todos los demás grupos, ya que tienen la puntuación más baja $(-0,3471)$. La puntuación más elevada es la del grupo con edades comprendidas entre los treinta y los cuarenta años $(0,2449)$, que se diferencia significativamente también de todos los demás grupos. No hay diferencias, sin embargo, entre los más jóvenes, de dieciocho a veintinueve años, y las personas entre cuarenta y cinco y cincuenta y nueve años (tabla 4). En el factor de "compra de productos de comercio justo», la edad se muestra linealmente asociada a este parámetro: a mayor edad, menor grado de compra de productos de este tipo, habiendo diferencias significativas entre todos los grupos comparados entre sí, excepto entre los dos segmentos más jóvenes (de dieciocho a veintinueve años frente a los de treinta a cuarenta y cuatro años) y los dos más mayores (de cuarenta y cinco a cincuenta y nueve años frente a mayores de sesenta años), en los que las diferencias no son significativas (tabla 5).

El análisis de varianza también nos indica que las diferencias existentes en los dos factores en función del nivel de estudios son estadísticamente signifi-

8. Para consultar las puntuaciones medias en los factores, véase la tabla incluida en el anexo final de este artículo. 
Tabla 4. Resultados del test de Tukey: factor de conocimiento del comercio justo según edad.

\begin{tabular}{lcccc}
\hline & 18-29 años & 30-44 años & 45-59 años & Más de 60 años \\
\hline 18-29 años & 1,000 & & & \\
30-44 años & $0,011^{*}$ & 1,000 & & \\
45-59 años & 0,885 ns & $0,001^{* *}$ & 1,000 & \\
Más de 60 años & $0,000^{* * *}$ & $0,000^{* * *}$ & $0,000^{* * *}$ & 1,000 \\
\hline
\end{tabular}

${ }^{* * *}(p<0,001) ;{ }^{* *}(p<0,01) ;^{*}(p<0,05) ;$ ns (no significativo).

Tabla 5. Resultados del test de Tukey: factor compra de productos de comercio justo según edad

\begin{tabular}{lcccc}
\hline & 18-29 años & 30-44 años & 45-59 años & Más de 60 años \\
\hline 18-29 años & 1,000 & & & \\
30-44 años & $0,865 \mathrm{~ns}$ & 1,000 & & \\
45-59 años & $0,002^{* *}$ & $0,019^{*}$ & 1,000 & \\
Más de 60 años & $0,000^{* * *}$ & $0,000^{* * *}$ & $0,162 \mathrm{~ns}$ & 1,000 \\
\hline
\end{tabular}

${ }^{* * *}(p<0,001) ;{ }^{* *}(p<0,01) ;^{*}(p<0,05) ;$ ns (no significativo).

Tabla 6. ANOVA unidireccional según estudios.

\begin{tabular}{lccccc}
\hline Factores & SC intergrupos & GL & MC & F & P \\
\hline Conocimiento del comercio justo & 317,155 & 4 & 79,289 & 89,970 & $0,000^{* * *}$ \\
Compra de comercio justo & 61,411 & 4 & 15,353 & 14,719 & $0,000^{* * *}$ \\
\hline
\end{tabular}

${ }^{* * *}(p<0,001) ;{ }^{* *}(p<0,01) ;^{*}(p<0,05) ;$ ns (no significativo).

cativas (tabla 6). La mayor puntuación en el factor de «conocimiento del comercio justo" se registra entre las personas con estudios universitarios $(0,6772)$ y la más reducida, en las personas con estudios primarios o menos $(-0,3239)$. Entre ambos extremos, la relación es lineal, de tal modo que, a mayor nivel de estudios, más elevada es la puntuación en el factor de "conocimiento de comercio justo». Pero, además, las diferencias según el nivel de estudios son significativamente diferentes cuando se compara a los diversos grupos entre sí por medio del test de Tukey, con la excepción de las personas con estudios de bachillerato y formación profesional, entre las cuales no existen diferencias estadísticamente significativas (tabla 7). En cuanto a la "compra de productos de comercio justo", también entre las personas con estudios universitarios se registra el nivel más alto y entre las personas con estudios primarios, el más bajo. Sin embargo, al comparar a los distintos grupos entre sí, las diferencias sólo son 
Tabla 7. Resultados del test de Tukey: factor conocimiento del comercio justo según estudios.

E. primarios $\mathrm{EGB} \quad \mathrm{FP} \quad$ Bachillerato Universitarios

\begin{tabular}{llllll}
\hline Estudios primarios & 1,000 & & & & \\
EGB o equivalente & $0,041^{*}$ & 1,000 & & & \\
Formación profesional & $0,000^{* * *}$ & $0,000^{* * *}$ & 1,000 & & \\
Bachillerato & $0,000^{* * *}$ & $0,000^{* * *}$ & 0,992 & 1,000 & \\
Universitarios & $0,000^{* * *}$ & $0,000^{* * *}$ & $0,000^{* * *}$ & $0,000^{* * *}$ & 1,000 \\
\hline
\end{tabular}

*** $(p<0,001)$; $^{* *}(p<0,01)$; $^{*}(p<0,05)$; ns (no significativo).

Tabla 8. Resultados del test de Tukey: factor compra de productos de comercio justo según estudios

\begin{tabular}{lcllcc}
\hline & E. primarios & EGB & FP & Bachillerato & Universitarios \\
\hline Estudios primarios & 1,000 & & & & \\
EGB o equivalente & $0,005^{* *}$ & 1,000 & & & \\
Formación profesional & $0,000^{* * *}$ & $0,657 \mathrm{~ns}$ & 1,000 & & \\
Bachillerato & $0,007^{* *}$ & $0,993 \mathrm{~ns}$ & $0,925 \mathrm{~ns}$ & 1,000 & \\
Universitarios & $0,000^{* * *}$ & $0,000^{* * *}$ & $0,153 \mathrm{~ns}$ & $0,014^{*}$ & 1,000 \\
\hline
\end{tabular}

*** $(p<0,001) ;^{* *}(p<0,01) ;^{*}(p<0,05)$; ns (no significativo).

significativas cuando se compara a los universitarios con los demás grupos (excepto con los de formación profesional) y a las personas con estudios primarios con todos los demás (tabla 8).

La situación laboral en que se encuentra el entrevistado también es una variable que registra diferencias significativas en los dos factores que estamos considerando (tabla 9). El conocimiento del comercio justo más elevado se da en el grupo de estudiantes $(0,2360)$, seguidos muy de cerca por las personas que trabajan $(0,1928)$, mientras las puntuaciones medias más reducidas son las de los jubilados $(-0,3178)$ y las personas dedicadas a las tareas domésticas $(-0,2546)$. El test de Tukey revela que las diferencias son estadísticamente significativas entre el grupo de trabajadores y estudiantes frente a jubilados y tareas domésticas, pero no dentro de cada uno de esos dos subgrupos (tabla 10). Por otro lado, las diferencias en la compra de productos de comercio justo únicamente son significativas cuando se compara a los jubilados (con $-0,1785$, la puntuación más baja) con los demás grupos (tabla 11).

Y, por último, examinamos la existencia de diferencias significativas debidas a la variable ocupación. El análisis de varianza nos indica que las diferencias sólo son significativas en "conocimiento del comercio justo» (tabla 12), 
Tabla 9. ANOVA unidireccional según situación laboral.

\begin{tabular}{lccccc}
\hline Factores & SC intergrupos & GL & MC & F & P \\
\hline Conocimiento del comercio justo & 135,389 & 4 & 33,847 & 35,712 & $0,000^{* * *}$ \\
Compra de comercio justo & 28,799 & 4 & 7,200 & 7,258 & $0,000^{* * *}$ \\
\hline
\end{tabular}

${ }^{* * *}(p<0,001) ;^{* *}(p<0,01) ;^{*}(p<0,05) ;$ ns (no significativo).

Tabla 10. Resultados del test de Tukey: factor de conocimiento del comercio justo según situación laboral.

\begin{tabular}{llllll}
\hline & Trabaja & Jubilado & Parados & Estudiantes & T. domésticas \\
\hline Trabaja & 1,000 & & & & \\
Jubilado & $0,000^{* * *}$ & 1,000 & & & \\
Parados & $0,564 \mathrm{~ns}$ & $0,000^{* * *}$ & 1,000 & & \\
Estudiantes & $0,981 \mathrm{~ns}$ & $0,000^{* * *}$ & $0,527 \mathrm{~ns}$ & 1,000 & \\
Tareas domésticas & $0,000^{* * *}$ & $0,858 \mathrm{~ns}$ & $0,001^{* *}$ & $0,000^{* * *}$ & 1,000 \\
\hline
\end{tabular}

${ }^{* * *}(p<0,001) ;{ }^{* *}(p<0,01) ;^{*}(p<0,05)$; ns (no significativo).

Tabla 11. Resultados del test de Tukey: factor de compra de comercio justo según situación laboral.

\begin{tabular}{lllllr}
\hline & Trabaja & Jubilado & Parados & Estudiantes & T. domésticas \\
\hline Trabaja & 1,000 & & & & \\
Jubilado & $0,000^{* * *}$ & 1,000 & & & \\
Parados & $0,999 \mathrm{~ns}$ & $0,100 \mathrm{~ns}$ & 1,000 & & \\
Estudiantes & $0,126 \mathrm{~ns}$ & $0,000^{* * *}$ & $0,253 \mathrm{~ns}$ & 1,000 & \\
Tareas domésticas & $0,985 \mathrm{~ns}$ & $0,029^{*}$ & $0,995 \mathrm{~ns}$ & $0,101 \mathrm{~ns}$ & 1,000 \\
\hline
\end{tabular}

${ }^{* * *}(p<0,001) ;{ }^{* *}(p<0,01) ;^{*}(p<0,05) ;$ ns (no significativo).

por lo que únicamente se ha aplicado el test de Tukey en ese factor. En todo caso, al comparar las puntuaciones en el citado factor según la situación ocupacional, sólo han resultado significativas las diferencias existentes entre los asalariados fijos y los asalariados eventuales, los primeros con una puntuación más elevada que los segundos (tabla 13).

Y si, hasta ahora, los análisis estadísticos realizados han tenido como objeto profundizar en el perfil sociodemográfico de la población española más próxima al comercio justo, a continuación se presentan los resultados de un análisis con el que se pretende abordar el segundo de los objetivos planteados al inicio de este trabajo: comprobar si, tal y como habitualmente se asume 
Tabla 12. ANOVA unidireccional según ocupación.

\begin{tabular}{lccccc}
\hline Factores & SC intergrupos & GL & MC & F & P \\
\hline Conocimiento del comercio justo & 16,371 & 3 & 5,457 & 5,494 & $0,001{ }^{* *}$ \\
Compra de comercio justo & 5,309 & 3 & 1,770 & 1,776 & $0,150 \mathrm{~ns}$ \\
\hline
\end{tabular}

${ }^{* * *}(p<0,001) ;^{* *}(p<0,01) ;^{*}(p<0,05) ;$ ns (no significativo).

Tabla 13. Resultados del test de Tukey: factor conocimiento del comercio justo según ocupación.

Asalariado fijo Asalariado eventual Empresario Autónomo

\begin{tabular}{lllll}
\hline Asalariado fijo & 1,000 & & & \\
Asalariado eventual & $0,001 * *$ & 1,000 & & \\
Empresario & $0,909 \mathrm{~ns}$ & $0,067 \mathrm{~ns}$ & 1,000 & \\
Autónomo & $0,244 \mathrm{~ns}$ & $0,641 \mathrm{~ns}$ & $0,362 \mathrm{~ns}$ & 1,000 \\
\hline
\end{tabular}

${ }^{* * *}(p<0,001) ;{ }^{* *}(p<0,01) ;{ }^{*}(p<0,05)$; ns (no significativo).

de modo implícito ${ }^{9}$, los consumidores de productos de comercio justo tienen una orientación global que determina en gran medida su relación con este tipo de campañas y productos, esto es, una preocupación por las condiciones en que se elaboran los productos que ellos consumen procedentes de otros países.

Para llevar a cabo esta tarea, hemos realizado dos análisis de regresión lineal múltiple en los que, obviamente, hemos incluido como variable dependiente los factores de "conocimiento" y "compra de productos de comercio justo». En cuanto a las variables independientes, hemos incluido, necesariamente, una variable que recogía el interés del entrevistado por las condiciones de fabricación o elaboración de los productos que adquiere procedentes de otros países (medido en una variable continua graduada en cuatro niveles: casi siempre, algunas veces, casi nunca y nunca); el autoposicionamiento ideológico (medido en una escala de 1 a 10 en la que el 1 se destinaba a las posiciones de extrema derecha y el 10, a las de extrema izquierda); la pertenencia a una ONG ( $1=$ sí, $0=$ no); el tamaño del hábitat (medido en una variable de siete niveles, de 1 = menos de 2.000 habitantes a 7 = más de un millón de habitantes). Junto a estas cuatro variables, hemos incluido el sexo ( 1 = varón, $0=$ mujer $)$; la edad; el nivel de estudios ( 1 = estudios primarios, 5 = estudios universitarios); y la situación laboral $(1=$ trabaja, $0=$ no trabaja). Hemos prescindido de la varia-

9. Al menos en la «literatura doctrinal» sobre comercio justo recogida en numerosas publicaciones de organizaciones dedicadas a éste, en la que se apela a la solidaridad internacional y a la necesidad de adoptar un punto de vista global, así como también en la imagen y en los eslóganes que suelen acompañar a este tipo de productos. 
Tabla 14. Análisis de regresión de los factores «conocimiento» $\mathrm{y}$ «compra de productos de comercio justo»

\begin{tabular}{|c|c|c|}
\hline & $\begin{array}{l}\text { Factor 1: conocimiento } \\
\text { del comercio justo }\end{array}$ & $\begin{array}{l}\text { Factor 2: compra } \\
\text { de productos } \\
\text { de comercio justo }\end{array}$ \\
\hline $\mathrm{R}$ & 0,382 & 0,250 \\
\hline $\mathrm{R}^{2}$ corregida & 0,142 & 0,058 \\
\hline F & 36,431 & 14,222 \\
\hline ANOVA (sig.) & 0,000 & 0,000 \\
\hline \multicolumn{3}{|c|}{ Coeficientes estandarizados Beta } \\
\hline (Constante) & $(* *)$ & $(*)$ \\
\hline $\begin{array}{l}\text { Interés condiciones } \\
\text { de producción }\end{array}$ & $0,162(* *)$ & $0,155\left(^{* *}\right)$ \\
\hline Tamaño de hábitat & $0,145\left(^{* *}\right)$ & 0,023 (ns) \\
\hline $\begin{array}{l}\text { Pertenencia a ONG } \\
(1=\text { Sí, } 0=\text { No })\end{array}$ & $0,129\left(^{* *}\right)$ & $0,080\left({ }^{* *}\right)$ \\
\hline Situación laboral activa & $0,101(* *)$ & $-0,002(\mathrm{~ns})$ \\
\hline Nivel de estudios & $0,089(* *)$ & 0,010 (ns) \\
\hline $\begin{array}{l}\text { Ideología } \\
(1 \text { = derecha, } 10 \text { = izquierda })\end{array}$ & $0,083(* *)$ & $0,053(*)$ \\
\hline Edad & $-0,069\left(^{* *}\right)$ & $-0,083(* *)$ \\
\hline Sexo $(1=$ varón, 0 = mujer $)$ & 0,035 (ns) & $-0,088(* *)$ \\
\hline
\end{tabular}

${ }^{* *}(p<0,01){ }^{*}(p<0,05) ; \mathrm{ns}(p>0,05)$.

ble ocupacional, habida cuenta del escasísimo carácter discriminante que mostraba cuando la estudiábamos por medio del análisis de varianza.

$\mathrm{Al}$ realizar la regresión (véase tabla 14), comprobamos que, respecto al factor de "conocimiento del comercio justo», el análisis resulta significativo, obteniéndose una $\mathrm{R}^{2}$ corregida del 14,2\%. Si examinamos los coeficientes estandarizados Beta, que nos muestran el peso de cada una de las variables independientes sobre la variable dependiente, vemos que precisamente el interés por las condiciones de producción registra el coeficiente estandarizado Beta más elevado $(0,162)$, lo que indica que a mayor interés por las condiciones de producción, mayor puntuación en el factor "conocimiento del comercio justo». La segunda variable que aparece en el análisis es el tamaño del hábitat $(0,145)$ que indicaría que, a medida que éste se incrementa, aumenta el conocimiento del comercio justo. A continuación, la pertenencia a una ONG $(0,129)$ y la situación laboral activa $(0,101)$ también tienen influencia sobre el factor de conocimiento. Las siguientes variables significativas que aparecen son el nivel de estudios $(0,089)$, la ideología $(0,083)$ y la edad del entrevistado $(-0,069)$. El análisis revela que a mayor nivel de estudios, a mayor cercanía a posiciones 
ideológicas de izquierda y a menor edad, mayor grado de conocimiento del comercio justo. Por último, la variable sexo no muestra una influencia estadísticamente significativa en el modelo.

En segundo lugar, la tabla 14 también recoge los resultados del análisis de regresión lineal múltiple para el factor de «compra de productos de comercio justo». En este caso, las variables independientes muestran una jerarquía claramente diferente al análisis del "conocimiento del comercio justo». Conviene resaltar y enfatizar, de cara al segundo de los objetivos de este trabajo, que la variable con mayor potencia explicativa vuelve a ser, de nuevo, el interés por las condiciones de producción $(0,155)$, lo que confirma la importancia de la orientación global en los consumidores de productos de comercio justo. La segunda variable que aparece en este análisis es, precisamente, la que se revelaba como no significativa en el factor de conocimiento, es decir, el sexo. El análisis de regresión muestra que el hecho de ser mujer tiene una influencia relevante sobre la compra de productos de comercio justo $(-0,088)$. A continuación, aparece la variable edad, lo que indica que, a menor edad, mayor comportamiento de compra de productos de comercio justo $(-0,083)$. Le sigue la pertenencia a una ONG $(0,080)$ y la cercanía a posiciones ideológicas de izquierdas $(0,053)$. Nótese, sin embargo, que, salvo en el caso de la variable referida al interés por las condiciones de producción, los coeficientes estandarizados Beta de este segundo análisis de regresión son inferiores a los que obteníamos en el anterior.

\section{Recapitulación y conclusiones}

La sociología del consumo ha señalado en los últimos años la aparición y desarrollo de una "ciudadanía a través del consumo" que se basaría en la incorporación de aspectos éticos como criterios de decisión de compra. Ésa sería una de las características básicas de los denominados «nuevos consumidores», sobre los cuales se ha afirmado que muestran una creciente sensibilidad en relación con sus actos de consumo y una toma de conciencia, no sólo en lo relativo a aspectos como el precio, la calidad y la facilidad de adquisición de los productos, sino también respecto al lugar y al modo cómo han sido fabricados, así también como a los actores que se benefician con su compra (Ray, 1999: 259; Burns, 1995). Según estos planteamientos, cada vez más, una importante porción de consumidores prefiere adquirir productos y marcas que se elaboran sin utilizar mano de obra infantil, sin condiciones de contratación injustas o sin salarios indignos y sin que peligre la seguridad, el puesto de trabajo o el medio ambiente (Barber, 2001).

Estas tendencias ponen de manifiesto tanto la multidimensionalidad del consumo como la obsolescencia de aquellos argumentos que lo convertían o en espacio de alienación o en ámbito de maximización racional de las preferencias individuales. Se estaría configurando, así, una nueva imagen del consumo, como espacio de elección limitada, pero responsable con el contexto social. Esto hace del consumidor un ser conectado a su entorno, del que obtie- 
ne percepciones, representaciones y valoraciones que se integran y se completan con el resto de sus ámbitos y esferas de actividad, y ello nos permite ver el proceso de consumo como «un conjunto de comportamientos que recogen y amplían, en el ámbito privado de los estilos de vida, los cambios civilizatorios y culturales de la sociedad en su conjunto» (Alonso, 2005). De este modo, el consumo se convierte progresivamente en espacio de recuperación y afirmación de la responsabilidad ciudadana. Aunque sus pautas de actuación no tienen nada que ver con el modelo de consumidor estrictamente racional al estilo del homo oeconomicus, la búsqueda de información y conocimiento, la reflexividad y la responsabilidad le conducen a hacer del consumo un ámbito de ciudadanía.

En este contexto cabe situar el origen y el desarrollo del comercio justo en las sociedades europeas actuales, como modalidad de producción y consumo que pretende impulsar un sistema alternativo de relaciones Norte-Sur mediante la promoción de cambios sociales y económicos, tanto en los países productores como en los consumidores. Respecto al primer tipo de países, trata de actuar sobre las transacciones comerciales, mientras que, respecto a los segundos, trata de cambiar las percepciones y los hábitos de sus ciudadanos. Por eso, en estos últimos países, el comercio justo implica la realización de tareas de sensibilización y denuncia que tienen su marco de acción en el espacio de la opinión pública. Desde ese espacio analítico, y tras revisar muy brevemente los principales hitos históricos y las dimensiones económicas del comercio justo en España, este trabajo ha tratado de identificar qué sector o grupos sociales se muestran más sensibles a las propuestas del comercio justo.

Los resultados empíricos de la investigación pivotan en torno a las dos dimensiones obtenidas mediante la aplicación de un análisis factorial de componentes principales a las variables con información relativa a comercio justo contenidas en una encuesta del Centro de Investigaciones Sociológicas de 2001. A partir de estos dos factores, "conocimiento" y "compra de productos de comercio justo", se ha examinado las diferencias existentes entre distintos grupos de población. Para ello, se ha realizado un análisis gráfico de las puntaciones medias en los factores y se han aplicado técnicas estadísticas para determinar la significatividad de las medias, como la prueba «t» de Student y el análisis de varianza unidireccional.

Estos análisis han mostrado la fuerte relación entre nivel de estudios y edad, tanto con el conocimiento como con la compra de productos de comercio justo, de manera que, a mayor nivel de estudios y a menor edad, mayor puntuación en los factores de conocimiento y compra de productos de comercio justo. Así, la población más joven, especialmente los menores de cuarenta y cuatro años y con estudios universitarios, constituirían los grupos sociales donde anidaría un mayor conocimiento e intención de compra de los productos de comercio justo. Otro hallazgo empírico de esta investigación es el referido a la situación laboral. Los análisis realizados han mostrado que la población que trabaja fuera del hogar, así como la que se encuentra cursando algún tipo de estudios, presenta las puntuaciones más elevadas en ambos fac- 
tores. Por el contrario, los grupos compuestos por personas dedicadas a las tareas domésticas y jubilados y pensionistas constituirían los sectores con los niveles más bajos de conocimiento y compra de productos de comercio justo.

La investigación realizada tenía un segundo objetivo, a saber, comprobar si, como habitualmente se insinúa, los individuos sensibilizados o consumidores de productos de comercio justo se caracterizan por una «orientación cognitiva global» mayor que la que presenta el resto de la población. Para abordar este segundo objetivo, se ha realizado un análisis de regresión lineal múltiple que ha confirmado la hipótesis inicial. La preocupación por las condiciones en que se elaboran los productos en otros países resultó ser la variable de mayor influencia, tanto en el factor de "conocimiento» como en el de "compra de productos de comercio justo", por encima de otras, como la pertenencia a una ONG, el autoposicionamiento ideológico en la escala izquierda-derecha y el tamaño del hábitat, así como también las referidas al sexo, edad, estudios y situación laboral del entrevistado.

En definitiva, estos resultados permiten afirmar que la «orientación global» constituye una característica del comercio justo (y, por tanto, de los «nuevos consumidores») que cabría añadir a las que ya han sido señaladas por otros autores, como la seguridad, el hedonismo, la simplificación, la individualización y el conocimiento (Alonso, 2005; Nodé-Langlois y Rizet, 1995; Ritzer, 2001). Estas características suponen la traslación al espacio social del consumo de tendencias que la teoría social contemporánea ha identificado como grandes transformaciones de la sociedad actual con respecto a la industrial (Beck, 1992, 1999, 2002; Beck, Giddens y Lash, 1994). Este último hallazgo podría ponerse en relación con la expansión de las capacidades de los individuos que, según Rosenau, propiciaría una emergencia de recursos cognitivos. Estos nuevos recursos estarían haciendo posible que los individuos tomen conciencia del origen de los problemas que les afectan, ya sean éstos de índole socioeconómica, ecológica o política (Rosenau, 1990: 335). Se trataría, pues, de una ampliación cognitiva que permitiría a los individuos comprender el entorno global que los rodea y generar juicios sobre el mismo (Teijo, 2002: 182). Todo ello podría estar produciendo un incremento de la conciencia de las posibilidades de actuar a escala global, y la conciencia de que los actos de cada individuo pueden tener repercusiones globales.

Habría que enfatizar, no obstante, la dificultad de realizar inferencias estadísticas estrictamente causales a partir de estos análisis, habida cuenta de la naturaleza exploratoria de esta investigación, tanto por lo que se refiere a la ausencia de un modelo teórico previo, como en lo que tiene que ver con las posibilidades analíticas ofrecidas por los datos disponibles. Serán necesarias, por tanto, investigaciones empíricas más específicas, tanto de naturaleza cualitativa como cualitativa, para determinar en qué consiste esa «orientación global» y cuáles son los mecanismos que la generan. En cualquier caso, y aunque con toda la prudencia necesaria, los resultados obtenidos en este trabajo permiten aventurar que esa «orientación global» constituye una característica del comercio justo y, por tanto, de los «nuevos consumidores». 
Por otro lado, los resultados de este trabajo vendrían a confirmar la progresiva transformación del consumo en una esfera de ciudadanía, una clara muestra de lo cual sería el fuerte impacto que la pertenencia a una ONG tiene tanto sobre el conocimiento del comercio justo como sobre la compra de este tipo de productos. En este sentido, puede afirmarse, por tanto, que el comercio justo, como movimiento social, muestra que el consumo no es algo ajeno a la ciudadanía y que tampoco la ciudadanía ha sido erradicada por el consumo, pues éste estaría conectando a los consumidores con responsabilidades políticas y sociales (Alonso, 2005). De manera que, en un contexto de creciente globalización, debilitamiento competencial de los estados nación y diseminación y ocultación del riesgo entre las redes de producción y distribución social, las acciones y opiniones de los consumidores exigiendo comportamientos cívicos y honestos estarían actuando como dispositivos de reflexividad y estarían abriendo el espacio social a nuevos ámbitos de participación social y exigencias de ciudadanía.

Habría que tener en cuenta, no obstante, que el proceso al que se acaba de hacer alusión no tiene una distribución social homogénea. De manera que, al hablar de la aparición de una "ciudadanía a través del consumo", no pueden pasar desapercibidas las diferencias sociales y la dualización de las culturas de consumo. Habría que enfatizar a ese respecto, por tanto, que, como se ha mostrado en la primera parte de la sección de resultados, esa «politización del consumo» se está produciendo en los grupos sociales con mayor capital simbólico y cultural, ya que son los sectores más dinámicos y con mayor nivel de estudios de la sociedad los que están desarrollando una «ciudadanía a través del consumo».

\section{Bibliografía}

AlOnSO, L. E. (2005). La era del consumo. Madrid: Siglo XXI.

BARBER, B. J. (2001). A place for us. Nueva York: Farrar / Pantheon Books.

BARRAT, M. (1998). Comercio justo, comercio injusto. Barcelona: Icaria.

BeCK, U. (1992). Risk Society: Towards a New Modernity. Londres: Sage.

- (1999). World Risk Society. Cambridge: Polity Press.

- (2002). Individualization. Londres: Sage.

BeCK, U.; Giddens, A.; Lash, S. (1994). Reflexive Modernization. Cambridge: Polity. BROWN, R. (1995). "Globalization and the national project». En: MACMILLAN, J.;

LinKLATER, A. (eds.). Boundaries in question. Londres: Pinter.

Burns, S. (1995). Fair Trade: A Rough Guide for Business. Twin.

CANTOS, E. (1998). El porqué del comercio justo. Hacia unas relaciones Norte-Sur más equitativas. Barcelona: Icaria.

Carraro, F.; Fernández, R.; Verdú, J. (2006). El rompecabezas de la equidad: Investigación y aportes críticos al Comercio Justo. Barcelona: Icaria.

EFTA (2001). Fair trade in Europe: Facts and Figures on the Fair in 18 European countries. Bruselas: European Fair Trade Association.

GIDDENS, A. (2001). Runaway world: how globalisation is reshaping our lives. Profile B. HinojosA, L. M. (2002). Comercio justo y derechos sociales. Madrid: Tecnos. 
InSTITUT CATALÀ DEL CONSUM (2004). Els catalans i el comerç just i solidari. Barcelona: DEP.

KeAne, J. (2003). «Reflexiones sobre la sociedad civil global». En: Vidal BenEyto, J. (dir.). Hacia una nueva sociedad civil desde la sociedad mundo. Madrid: Taurus.

KRIER, J.-M. (2001). Fair Trade in Europe 2001. EFTA.

MartíneZ-Orozco, S. (1996). Comerç just, consum responsable. Barcelona: Octaedro. Matathia, I.; Salzman, M. (2001). Tendencias: Estilos de vida para el nuevo milenio. Barcelona: Planeta.

MONTAGUT, X.; VIVAS, E. (2006). ¿Adónde va el comercio justo? Barcelona: Icaria.

Morace F. (1990). Controtendenze. Milán: Domus Academy.

NodÉ-Langlois, N.; Rizet, L. (1995). La consommation. París: Le Monde Éditions.

RaY, C. (1999). «Endogenous Development in the Era of Reflexive Modernity». Journal of Rural Studies, vol. 15, no 3, p. 257-267.

RitZER, G. (2001). Explorations in the Sociology of Consumption. Londres: Sage.

Robertson, R. (1992). Globalization: Social Theory and Global Culture. Londres: Sage.

ROCHEFORT, R. (1996). La société des consommateurs. París: O. Jacob.

Rosenau, J. N. (1990). Turbulence in world politics: a theory of change and continuity. Princeton: Princeton University Press.

Setem (1999). Café amargo. Por un comercio Norte-Sur más justo. Barcelona: Icaria.

- (2004). El comercio justo en España: Situación y perspectivas. Barcelona: Icaria. SOLÉ, E. (2003). Qué es el comercio justo. Barcelona: Integral.

TEIJO, C. (2002). «Redes transnacionales de participación ciudadana y ONG. Alcance y sentido de la sociedad civil internacional». En: REVILLA, M. (ed.). Las ONG y la política. Madrid: Ediciones Istmo.

WATERS, M. (1995). Globalization. Londres: Routledge. 


\section{Anexo}

Puntuaciones medias en los factores según variables sociodemográficas.

\begin{tabular}{llcc}
\hline & & $\begin{array}{c}\text { Conocimiento } \\
\text { del comercio justo }\end{array}$ & $\begin{array}{l}\text { Compra } \\
\text { de productos } \\
\text { de comercio justo }\end{array}$ \\
\hline Sexo & Hombre & 0,0848 & $-0,0683$ \\
Edad & Mujer & $-0,0793$ & 0,0639 \\
& $18-29$ años & 0,0766 & 0,1456 \\
Estudios & 30-44 años & 0,2449 & 0,1025 \\
& 45-59 años & 0,0340 & $-0,0637$ \\
& Más de 60 años & $-0,3471$ & $-0,1831$ \\
& Estudios primarios & $-0,3239$ & $-0,1822$ \\
& EGB & $-0,1754$ & 0,0187 \\
& Formación profesional & 0,2606 & 0,1169 \\
& Bachillerato & 0,3231 & 0,0498 \\
& Universitarios & 0,6772 & 0,2944 \\
& Trabaja & 0,1928 & 0,0410 \\
& Jubilado / pensionista & $-0,3178$ & $-0,1785$ \\
& Parados & 0,0821 & 0,0228 \\
& Estudiantes & 0,2360 & 0,2284 \\
& Tareas domésticas & $-0,2546$ & 0,0113 \\
& Asalariado fijo & 0,0567 & $-0,0077$ \\
& Asalariado eventual o interino & $-0,1250$ & 0,0648 \\
& Empresario & 0,1203 & $-0,0531$ \\
& Trabajador autónomo & $-0,0493$ & $-0,0776$ \\
\hline
\end{tabular}

